\title{
Recent Developments in the Prevention and Treatment of Missing Data
}

Therapeutic Innovation \& Regulatory Science 00(0) $1-13$

(C) The Author(s) 2013

Reprints and permission:

sagepub.com/journalsPermissions.nav DOI: $10.1177 / 2168479013501310$

tirs.sagepub.com

\author{
C. Mallinckrodt, $\mathrm{PhD}^{\prime}$, J. Roger, $\mathrm{PhD}^{2}$, C. Chuang-Stein, $\mathbf{P h D}^{3}$, \\ G. Molenberghs, $\mathrm{PhD}^{4,5}$, M. O'Kelly, $\mathrm{PhD}^{6}$, B. Ratitch, $\mathrm{PhD}^{7}$, \\ M. Janssens, $M^{4,5}$, and $P$. Bunouf, PhD $^{8}$
}

\begin{abstract}
Recent research has fostered new guidance on preventing and treating missing data, most notably the landmark expert panel report from the National Research Council (NRC) that was commissioned by FDA. One of the findings from that panel was the need for better software tools to conduct missing data sensitivity analyses and frameworks for drawing inference from them. In response to the NRC recommendations, a Scientific Working Group was formed under the Auspices of the Drug Information Association (DIASWG). The present paper is from work of the DIASWG. Specifically, the NRC panel's 18 recommendations are distilled into 3 pillars for dealing with missing data: (I) providing clearly stated objectives and causal estimands; (2) preventing as much missing data as possible; and (3) combining a sensible primary analysis with sensitivity analyses to assess robustness of inferences to missing data assumptions. Sample data sets are used to illustrate how sensitivity analyses can be used to assess robustness of inferences to missing data assumptions. The suite of software tools used to conduct the sensitivity analyses are freely available for public use at www.missingdata.org.uk.
\end{abstract}

\section{Keywords}

missing data, clinical trials, sensitivity analyses

\section{Introduction}

Missing data pose an ever-present problem in clinical trials that can bias treatment group comparisons and inflate rates of falsenegative and false-positive results. ${ }^{1-5}$ Until recently, guidelines for analyzing clinical trial data provided only limited advice on how to handle missing data. Analyses tended to be simple and ad hoc, with little forethought given to preventing missing data. The motivation for dealing with missing data may have been as much to foster computational feasibility in an era of limited computing power as to counteract the potential bias from the missing values. ${ }^{5}$

For example, complete case analyses include only cases for which all measurements were recorded, yielding the data structure that would have resulted with no missing data. Therefore, standard software and simple statistical analyses can be used. Unfortunately, the loss of information is usually substantial, and severe bias can result when the outcomes for patients who discontinue differ from those who complete. ${ }^{5}$ Alternative means to obtain complete data sets were based on imputing (explicitly filling in) the missing data. However, the simple imputation strategies such as baseline and last observation carried forward (BOCF, LOCF) that were used widely in clinical trials also have serious drawbacks. These methods entail restrictive assumptions that are unlikely to hold, and the uncertainty of imputation is not taken into account as imputed values are not distinguished from observed values. Therefore, biased estimates of treatment effects and inflated rates of falsepositive and false-negative results are likely. ${ }^{1,3,5,6}$

However, the use of simple methods set a historical precedent that, when combined with the desire to compare results with historical findings and the belief that LOCF and BOCF

\footnotetext{
' Lilly Research Labs, Eli Lilly and Co, Indianapolis, IN, USA

${ }^{2}$ London School of Hygiene and Tropical Medicine, London, UK

${ }^{3}$ Pfizer Inc, Kalamazoo, MI, USA

${ }^{4}$ I-BioStat, Hasselt University, Diepenbeek, Belgium

${ }^{5}$ I-BioStat, Katholieke Universiteit, Leuven, Belgium

${ }^{6}$ Quintiles, Fairview, Dublin, Ireland

${ }^{7}$ Quintiles, Saint-Laurent, Québec, Canada

${ }^{8}$ Laboratoires Pierre Fabre, Toulouse, France
}

Submitted I5-Apr-20I3; accepted 23-Jul-20I3

Corresponding Author:

C. Mallinckrodt, Lilly Research Labs, Eli Lilly and Co, Indianapolis, IN 46285, USA. Email: cmallinc@lilly.com 
yielded conservative estimates of treatment effects, fostered continued use of these methods. "Conservative" in this context was often interpreted as underestimating the magnitude of a treatment's benefit (efficacy), thereby providing additional protection against erroneous approval of ineffective interventions. While potentially appealing in spirit, conservatism is difficult to achieve in practice because underestimating a treatment effect, while conservative in a superiority test, could be anticonservative in a noninferiority test and would certainly be anticonservative for testing safety outcomes. Moreover, the anticipated conditions that would render an estimate conservative frequently are not realized in actual practice. ${ }^{6}$

Fortunately, the problem of missing data has been an active area of investigation, and there have been many advances in statistical theory and in our ability to implement that theory. ${ }^{1}$ This research set the stage for new and updated guidance for preventing and handling missing data in clinical trials. For example, new guidance was released by the European Medicines Agency (EMA), ${ }^{2}$ an expert panel from the National Research Council (NRC) that was commissioned by the Food and Drug Administration (FDA) issued an extensive set of recommendations, ${ }^{3}$ two senior leaders at FDA published their thoughts on the NRC recommendations, ${ }^{4}$ and entire textbooks have been devoted to missing data in clinical trials. ${ }^{1,5}$ The present paper distills, discusses, and illustrates key themes from the NRC report and makes freely available for public use customized software tools for implementing sensitivity analyses.

\section{National Research Council Recommendations}

At the request of and with funding from FDA, an expert panel was created by the NRC's Committee on National Statistics. The panel included approximately 15 members, mostly statisticians with academic affiliations and experience in clinical trials. The panel interviewed prominent statisticians in the pharmaceutical industry and FDA. Information from these interviews and the panel's experience formed the basis of their 144-page report, which was published in $2010 .^{3}$ This report is clearly influencing practice. Regulators are commonly asking drug development teams to use the recommendations when proposing and implementing plans to deal with missing data.

Although the panel focused on phase III trials, the recommendations are useful regardless of stage of development. The recommendations set forth an overarching framework for tackling the problem of missing data. Key pillars of this framework include (1) providing precise and clear specification of trial objectives; (2) designing and conducting trials in a way to maximize patient retention; and (3) including a sensible primary analysis and sensitivity analyses that support the research question and assess robustness of the primary result to missing data assumptions.

\section{Pillar I: Setting Clear and Precise Objectives}

An important evolution in discussions about missing data has been the focus on clarity of objectives. In fact, the first recommendation from the NRC guidance ${ }^{3}$ was that the objectives be clearly specified. The need for clarity is driven by ambiguities arising from the missing data. Data may be intermittently missing or missing because of dropout. Patients may or may not be given rescue medications. Assessments after withdrawal from the initially randomized medication or after the addition of rescue medications may or may not be made and, if made, may or may not be included in the primary analysis. ${ }^{7}$

For clarity the following distinction is made. Patient dropout occurs when the patient discontinues the initially randomized medication and no further observations are taken. Analysis dropout occurs when patients deviate from the originally randomized treatment regime (stop medication and/or add rescue medication) and observations are made but are not included in the analysis. Whether or not follow-up data are included in the analysis depends on the estimand. Within a single study, some analyses may include the follow-up data and others may not.

Conceptually, an estimand is simply what is being estimated. Components of estimands for longitudinal trials include the parameter (eg, difference between treatments in mean change), time point or duration of exposure (eg, at week 8), outcome measure (eg, diastolic blood pressure), population (eg, in patients diagnosed with hypertension), and inclusion or exclusion of follow-up data after discontinuation of the originally assigned study medication and/or initiation of rescue medication. ${ }^{1}$

Much of the debate on appropriate estimands centers on whether the focus is on efficacy or effectiveness. Efficacy may be viewed as the effects of the drug if taken as directed: for example, the benefit of the drug expected at the end point of the trial in patients who took the drug as directed. This has also been referred to as a per-protocol estimand. Effectiveness may be viewed as the effects of the drug as actually taken and has also been referred to as an intention-to-treat (ITT) estimand. ${ }^{1,6}$ Choice of estimand drives the decision about whether followup data are included in an analysis.

Although a full discussion of estimands for longitudinal trials and the data and analyses needed to estimate them is beyond the present scope, many of the key issues are embedded within the subset of estimands listed in Table 1 and described next. ${ }^{8}$

\section{A. Difference in outcome improvement at the planned end point for all randomized participants. Follow-up data (after withdrawal of initially randomized medication and/or initiation of rescue medication) are included in the analysis.}


Table I. Estimands and their key attributes.

\begin{tabular}{|c|c|c|c|c|c|}
\hline Estimand & Hypothesis & Inference & Population & End Point & $\begin{array}{l}\text { Use of Data After Withdrawal of } \\
\text { Randomized Study Medication }\end{array}$ \\
\hline A & Effectiveness & Treatment policy & All patients & Planned end point & Included in primary analysis \\
\hline C & Effectiveness & Initially randomized medication & All patients & Planned end point & Likely imputed \\
\hline
\end{tabular}

This estimand compares groups regardless of what treatment participants actually received, and therefore inference is on the effectiveness of the treatment regimen, not the originally randomized treatments.

B. Difference in outcome improvement if all patients adhered. Follow-up data are not included in the analysis. This estimand compares the causal effects of the initially randomized drugs if taken as directed - an efficacy estimand.

C. Difference in outcome improvement in all randomized patients at the planned end point of the trial attributable to the initially randomized medication. This estimand assesses effectiveness at the planned end point, focusing on the causal effects attributable to the initially randomized medications.

Conceptually, estimands $\mathrm{A}$ and $\mathrm{C}$ require follow-up data. The intent of estimand $\mathrm{C}$ is to avoid the confounding effects of rescue medications and draw inference to the originally randomized treatments. Estimand $\mathrm{B}$ and estimand $\mathrm{C}$ both focus on causal effects of the initially randomized medications, in all randomized patients, at the planned end point of the trial. Estimand $\mathrm{B}$ focuses on what to expect when patients adhere to treatment, and estimand $\mathrm{C}$ focuses on what was actually observed. Estimand B assesses efficacy and estimand C assesses effectiveness.

Estimands B and C can be used in combination as the primary and secondary estimands, an approach particularly useful in trials assessing symptomatic treatments. For example, in a proof-of-concept study the focus may be primarily on efficacy, but as development progresses the focus may shift toward effectiveness if the study conditions are naturalistic enough to be generalized to clinical practice. ${ }^{1}$

Each estimand has strengths and limitations. Estimand A may closely mirror clinical practice. But it tests hypotheses about treatment policies, and the most relevant research questions in confirmatory or earlier clinical trials are often about the causal effects of the investigational drugs, not treatment policies. In the intention-to-treat (ITT) framework where inference is drawn based on the originally assigned treatments, the inclusion of follow-up data when rescue medications are allowed can mask or exaggerate both the efficacy and safety effects of the initially assigned treatments, thereby invalidating causal inferences for the originally assigned medication. ${ }^{1,7}$
Estimand B assesses what happens if a drug is taken as directed, which is useful because the majority of patients do adhere. However, on a group-aggregate basis, estimand B is hypothetical because there will always be some patients who do not adhere. ${ }^{3}$ Estimand $\mathrm{C}$ focuses on effectiveness of the initially randomized drug, free of the confounding effects of rescue medications. However, ethical considerations often mandate that rescue medications be allowed after patients discontinue randomized study medication.

Given the confounding effects of rescue medications and the ethical need to allow them, one approach to assessing effectiveness is to impute the data after discontinuation of the initially randomized study medication assuming that initially randomized active medications have no effect or a diminishing effect after discontinuation. This assumption is often reasonable in trials of symptomatic interventions. ${ }^{4}$ Controlled imputation analyses, discussed in a subsequent section, are a potential means to impute the follow-up data needed to estimate estimand C. ${ }^{1,9}$

\section{Pillar 2: Minimize Missing Data}

Despite advantages of newer analytic methods, an important conundrum inherent to all analyses of incomplete data remains. Namely, all analyses require assumptions about the missing data, but the data about which the assumptions are made are missing. Hence, the assumptions cannot be verified from the data, and the appropriateness of analyses and inference cannot be ensured. ${ }^{10}$ Therefore, minimizing missing data is the best means of dealing with the problem. ${ }^{1,3,11}$

The NRC recommendations ${ }^{3}$ detail a number of trial design and conduct options for maximizing retention on the originally randomized study drugs. A detailed discussion of these approaches is beyond our present scope but is available elsewhere. ${ }^{1}$ Key elements of those discussions include that while design options may lower dropout, the impact that these options have on other aspects of the trial must be considered. Some design options may improve retention for patients randomized to active medications but not to placebo or active control.

Some of the trial design options noted in the NRC guidance ${ }^{3}$ included enrolling a target subpopulation for whom the riskbenefit of the drug is more favorable, or identifying such 
subgroups during the course of the trial via enrichment or runin designs. However, examples where this has been done successfully in the context of lowering rates of dropout are rare. Other design options in the NRC guidance included use of add-on designs and flexible dosing.

Specific guidance on trial conduct from the NRC panel included minimizing patient burden, using efficient data capture procedures, providing education on the importance of complete data, and monitoring and providing incentives for complete data. ${ }^{3}$ Simply put, lowering rates of dropout can be as much about behavior as about design and process.

\section{Pillar 3: Appropriate Primary and Sensitivity Analyses}

Pillar 3 includes 2 equally important parts: an appropriate primary analysis and sensitivity analyses to assess the robustness of results to key assumptions of the primary analysis. Both of these are necessary because despite all efforts to minimize missing data, anticipating complete data is not realistic.

To choose an appropriate primary analysis, the mechanisms leading to the missingness must be considered. Data are missing completely at random (MCAR) if, conditional upon the independent variables in the analysis, the probability of missingness does not depend on either the observed or unobserved outcomes of the variable being analyzed (dependent variable). In practical terms, MCAR is when the variable being analyzed is not related to the likelihood of missing data. If the variable being analyzed is an efficacy outcome, MCAR is akin to believing that efficacy does not influence the probability of dropout.

Data are missing at random (MAR) if, conditional upon the independent variables in the analysis and the observed outcomes of the dependent variable, the probability of missingness does not depend on the unobserved outcomes of the dependent variable. Therefore, MAR is less restrictive than MCAR because MAR allows the observed outcomes of the variable being analyzed to influence the probability of dropout. In practical terms, MAR is when the observed values of the variable being analyzed along with the independent variables in the analysis explain why data are missing and the unobserved data contain no additional information.

Data are missing not at random (MNAR) if the unobserved data contain information not foreseen by the observed data such that the unobserved data cannot be projected or estimated from the observed outcomes. More formally, MNAR is when, conditional upon the independent variables in the analysis model and the observed outcomes of the dependent variable, the probability of missingness does depend on the unobserved outcomes of the variable being analyzed. Another useful way to think about MNAR is that if, conditional upon observed outcomes, the statistical behavior (means, variances, etc) of the unobserved data is the same as if the data had been observed, then the missingness is MAR; if not, then it is MNAR. ${ }^{1,3,5,9}$

In longitudinal clinical trials, MCAR is unlikely to be valid; MAR is often plausible but never provable; and going beyond MAR to MNAR requires assumptions that are not testable. Hence, no single MNAR analysis can be definitive. ${ }^{3,10}$ In clinical trial settings, a primary analysis based on MAR is often reasonable, whereas complete case and single imputation methods that require MCAR and/or other restrictive assumptions are usually not reasonable. ${ }^{1,3,5,6}$ An MAR analysis may be especially reasonable when combined with rigorous efforts to maximize retention on the initially randomized medications. ${ }^{1}$

Methods common in the statistical literature based on MAR include likelihood-based analyses, multiple imputation (MI), and weighted generalized estimating equations (wGEE). These MAR methods use the observed data to model or impute the missing data. In multiple imputation, a model is used to explicitly estimate from the observed data what the missing data would have been had they been observed; then the imputed missing values and actually observed values are combined and analyzed as if complete. This process is repeated multiple times to foster assessment of uncertainty arising from imputing the missing values. In likelihood-based analyses and wGEE, missing values are not explicitly imputed. However, the analyses are formulated such that the incompleteness is taken directly into account via the analysis models. The specific attributes of each method can be used to tailor an analysis to the situation at hand. Detailed descriptions and applications of these methods can be found elsewhere. ${ }^{5}$

The focus of sensitivity analyses is to assess robustness of the primary analysis to assumptions. Therefore, appropriate sensitivity analyses will depend on the estimand and the analysis. With an MAR primary analysis, assessing robustness of conclusions to departures from MAR should be a focus of sensitivity analyses. This can be accomplished by implementing a series of plausible MNAR analyses and assessing consistency of results. ${ }^{1,3,5,6,9,10}$ Three common families of MNAR analyses are shared-parameter models, pattern-mixture models, and selection models. Detailed descriptions and applications of these methods can be found elsewhere. ${ }^{5}$

Selection models are multivariate models for repeated measures where one variable is the efficacy outcome from the primary analysis and the second is the repeated binary outcome for dropout that is modeled via logistic regression. The 2 parts of the selection model are often referred to as the measurement model and the dropout model. The 2 models are linked in that the dependent variable from the measurement model is a predictor (independent) variable in the dropout model. Shared-parameter models can also be thought of as multivariate models, where one variable is again the continuous efficacy outcome from the primary analysis and the second variable is time to dropout. The 
measurement and dropout models are linked by a set of latent variables, latent classes, and/or random effects that are assumed to influence both the outcome variable and time to dropout. ${ }^{1,5,10}$ These models are fitted either using maximum likelihood or as Bayesian models using data augmentation and Markov chain Monte Carlo (MCMC) techniques.

Pattern-mixture models fit a separate response model for each pattern of missing values weighted by their respective probabilities, and outcomes are combined across patterns. Patterns are often defined by time of dropout but could be defined by reason for discontinuation or other means. Pattern-mixture models are often by construction underidentified, that is, overspecified. For example, assume that the goal is to estimate the difference between treatments at end point and 3 dropout patterns are used: early dropouts, late dropouts, and completers. Missing end point values for the early and late dropout groups must be imputed; however, information must be borrowed from other groups because there is no information about end point values in the early and late dropout patterns.

This problem can be resolved through the use of identifying restrictions where inestimable parameters of the incomplete patterns are set equal to (functions of) those of other patterns. Three common identifying restrictions are (1) complete case missing values (CCMV), where information is borrowed from the completers; (2) neighboring case missing values (NCMV), where information is borrowed from the nearest identified pattern; and (3) available case missing values (ACMV), where information is borrowed from all patterns where the information is available. ${ }^{5}$

In addition, a general family of restrictions can be defined as non-future dependent missing value restrictions (NFD), where one conditional distribution per incomplete pattern is left unidentified. In other words, the distribution of the "current" unobserved measurement given the previous measurements is unconstrained. In practice, this can be accomplished by using data only up to and including the time point being imputed as the basis for estimating parameters for the imputation model. ${ }^{5}$ When information is borrowed in the NFD family via CCMV or NCMV, the mechanism is MNAR. The ACMV restriction assumes MAR. Therefore, comparing results from ACMV with NFD-CCMV or NFD-NCMV assesses sensitivity of results to departures from MAR. ${ }^{5}$

Recently, another family of methods referred to as controlled imputation has seen increasing discussion in the literature and use in practice. ${ }^{9,12-16}$ Controlled imputation approaches are specific formulations of pattern-mixture models whose basic idea is to construct a principled set of imputations that exhibit a specific departure from MAR.

Multiple imputation has been typically implemented assuming MAR with separate imputation models for the drug and placebo (control) arms (in a 2-arm study). For MNAR analyses that exhibit a specific and relevant departure from MAR, one subfamily of approaches within controlled imputation, referred to as reference-based imputation, uses one imputation model that is derived from data in the reference (eg, placebo, or standard of care) group but applies that model to both the drug and placebo (reference) arms. ${ }^{1,9,14,15}$

Using one imputation model for both treatment arms generally diminishes the difference between the arms compared with MAR approaches that use separate imputation models for each arm. The intent is to generate a plausibly conservative estimate of an efficacy estimand that can be used to define a worst plausible result. After the missing values are imputed, the primary analysis is repeated. If inferences agree with the primary result, the findings are robust to the plausible departures from MAR. Alternatively, the same result can be interpreted as an estimate of effectiveness that reflects a change in or discontinuation of treatment. ${ }^{1}$

Reference-based imputations can be tailored to specific clinical scenarios. One variant of reference-based imputation, termed jump to reference ( $\mathrm{J} 2 \mathrm{R})$, is implemented such that imputed values for patients who discontinue the active arm immediately take on the attributes of the reference arm (placebo). That is, the treatment benefit in patients who discontinue the active arm disappears immediately upon discontinuation. The J2R approach would therefore be useful for symptomatic treatments with short duration of action. In the copy reference (CR) method, the imputations result in a treatment effect that diminishes after dropout in accordance with the correlation structure implied by the imputation model. The CR approach would therefore be useful for symptomatic treatments with long duration of action. The copy increment method (CIR) maintains the treatment effect after discontinuation but matches changes after withdrawal to changes in the reference arm and would therefore be appropriate for treatments thought to alter the underlying disease process (disease modification). ${ }^{9}$

Controlled imputation can also be used to assess sensitivity by repeatedly adjusting the imputations to provide a progressively more severe stress test to assess how extreme the departures from MAR must be to overturn the primary result. ${ }^{14,16}$ Typically, only imputed values for the experimental arm are adjusted while the control arm is handled with an MARbased approach. The basic idea is to impute the missing values and subtract a value (delta) from the imputed values of the experimental arm. Two distinct implementations exist. One method uses the sequential regression approach to MI and adds delta to the imputed value, so that it feeds through in the imputation process to later visits. In the second approach, delta is added to the imputed value for that visit only and does not feed through in this way. In some interpretations of the first approach, delta is only applied to the first visit after withdrawal, similar in concept to NFD. 
Table 2. Week of last visit (no. of subjects).

\begin{tabular}{|c|c|c|c|c|c|c|c|c|c|c|}
\hline & \multicolumn{5}{|c|}{ High Dropout } & \multicolumn{5}{|c|}{ Low Dropout } \\
\hline Drug & 9 & 6 & 10 & 5 & 70 & 2 & 3 & 2 & I & 92 \\
\hline
\end{tabular}

The basic concept is to repeat the primary analysis on the delta-adjusted data set to see whether the conclusion of the primary analysis is overturned. If not, a larger delta is chosen and the process repeated until the primary result is overturned. If the delta required to overturn the primary result is not a plausible departure from MAR, then the primary result is robust to plausible departures from MAR. As with reference-based imputation, delta-adjustment methods can be tailored to specific situations. ${ }^{3,14,16}$

\section{Example}

The following example illustrates the 3 pillars of dealing with missing data: clearly stating objectives and estimands, preventing missing data, and conducting a reasonable primary analysis that is supported by sensitivity analyses for key missing data assumptions. The example is not intended to be a fully comprehensive assessment of sensitivity but rather serves as an illustration of selected points. Full details of the analyses are not presented here. The programs used to analyze the sample data and other software tools for sensitivity analyses are freely available at www.missingdata.org.uk.

The data sets used in this example were somewhat contrived to avoid implications for marketed drugs. Nevertheless, the key features of the original data were preserved. The original data were from 2 nearly identically designed antidepressant clinical trials that were originally reported by Goldstein et al $^{17}$ and Detke et al. ${ }^{18}$ Each trial had 4 treatment arms with approximately 90 patients each that included 2 doses of an experimental medication (subsequently granted marketing authorizations in most major jurisdictions), an approved medication, and placebo. Assessments on the Hamilton 17-item rating scale for depression $\left(\mathrm{HAMD}_{17}\right)^{19}$ were taken at baseline and weeks 1, 2, 4, 6, and 8 in each trial. All patients from the original placebo arm were included along with a contrived drug arm that was created by randomly selecting patients from the nonplacebo arms. Random selection continued until 100 drug-treated patients were selected. In addition to all the original placebo-treated patients, additional placebo-treated patients were randomly reselected so that there were also 100 patients in the contrived placebo arms. For these reselected placebo-treated patients, a new patient identification number was assigned and outcomes were adjusted to create new observations by adding a randomly generated value to each patient's observations.

These trials are referred to as the low and high dropout data sets. In the high dropout data set, completion rates were $70 \%$ for drug and $60 \%$ for placebo (Table 2). In the low dropout data set, completion rates were $92 \%$ in both the drug and placebo arms. The dropout rates in the contrived data sets closely mirrored those in the corresponding original studies. The design difference that may explain the difference in dropout rates between these two otherwise similar trials was that the low dropout data set came from a study conducted in Eastern Europe that included a 6-month extension treatment period after the 8-week acute treatment phase and used titration dosing. The high dropout data set came from a study conducted in the US that did not have the extension treatment period and used fixed dosing.

Visit-wise mean changes for patients who completed the trials versus those who discontinued early are summarized in Figures 1 and 2 for the low and high dropout data sets, respectively. In the high dropout data set, patients who discontinued early had smaller average improvement than completers, suggesting that missing data did not arise from an MCAR mechanism. With only a few dropouts at each visit in the low dropout data set, trends were not readily identifiable.

The primary analytic objective for each data set was to compare the efficacy (benefit if taken as directed) of drug versus placebo. The primary estimand was the difference between treatments in mean change from baseline to week 8, expressed as Placebo - Drug, so that resulting values are usually positive, with larger values indicating greater improvement. The primary analysis used a restricted maximum likelihood (REML)-based repeated-measures approach. The analyses included the fixed, categorical effects of treatment, investigative site, visit, and treatment-by-visit interaction as well as the continuous, fixed covariates of baseline score and baseline score-by-visit interaction. An unstructured (co)variance structure shared across treatment groups was used to model the within-patient errors. The Kenward-Roger approximation was used to estimate denominator degrees of freedom and adjust standard errors. Analyses were implemented with SAS PROC MIXED. ${ }^{20}$ The primary comparison was the contrast (difference in least squares mean [LSMEAN]) between treatments at the last visit (week 8). LSMEANs were evaluated at the mean baseline across subjects rather than the default mean 


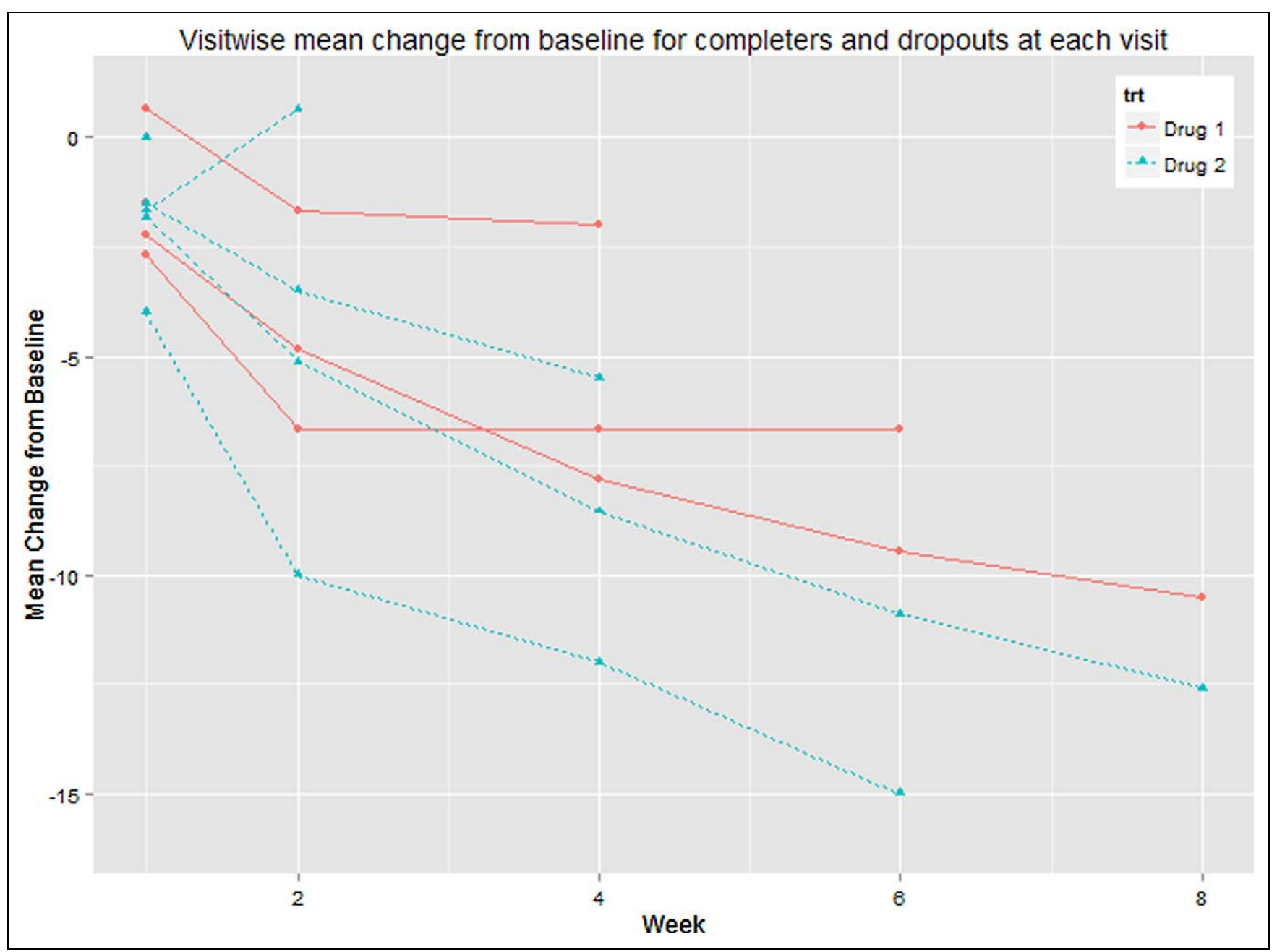

Figure I. Visit-wise mean changes from baseline in $\mathrm{HAMD}_{17}$ for patients who completed the trial versus those who discontinued early in the low dropout data set.

across data records, while the default equal spread across the levels for pooled investigator was maintained.

This slightly unusual but recommended approach has no impact on the difference between treatment LSMEAN, which is important for study interpretation. By causing the predicted values for each arm to be evaluated under identical situations, we can unambiguously compare the ways that the different methods handle the missing data in each group. The missing data assumption for the primary analysis was that missing data arose from an MAR mechanism. The estimand is a de jure or efficacy one, as subjects who withdraw are treated as if they are the same as those remaining on randomized treatment within the study. Sensitivity analyses to assess the consequences of departures from MAR on inferences while continuing to answer the same estimand included selection and pattern mixture models. An alternative de facto or effectiveness estimand, where subjects are assumed to revert to placebo following withdrawal, was addressed with reference-based imputation. Some delta-adjusted controlled imputation approaches are also included where the estimand is less clear.

Results from the primary analyses are summarized in Tables 3 and 4. In the high dropout data set, the advantage of drug over placebo in mean change from baseline to week 8 was 2.37 (standard error $[\mathrm{SE}]=1.03, P=.024$ ). This precision is equivalent that of 74 completers per arm. The corresponding values in the low dropout data set were $1.86(\mathrm{SE}=0.70$, $P=.009$ ). The standard error for the difference in LSMEAN at week 8 in the high dropout data set was $47 \%$ larger than in the low dropout data set. This was partly due to the lower variability in the low dropout than in the high dropout data set (Table 4). The larger variability in the high dropout group would have led to an increased standard error of $32 \%$ on its own. The additional variability in the high dropout set comes from the additional quantity of missing data.

This model assumed that the pooled investigator effect was the same at every week. Some argue that all covariates should 


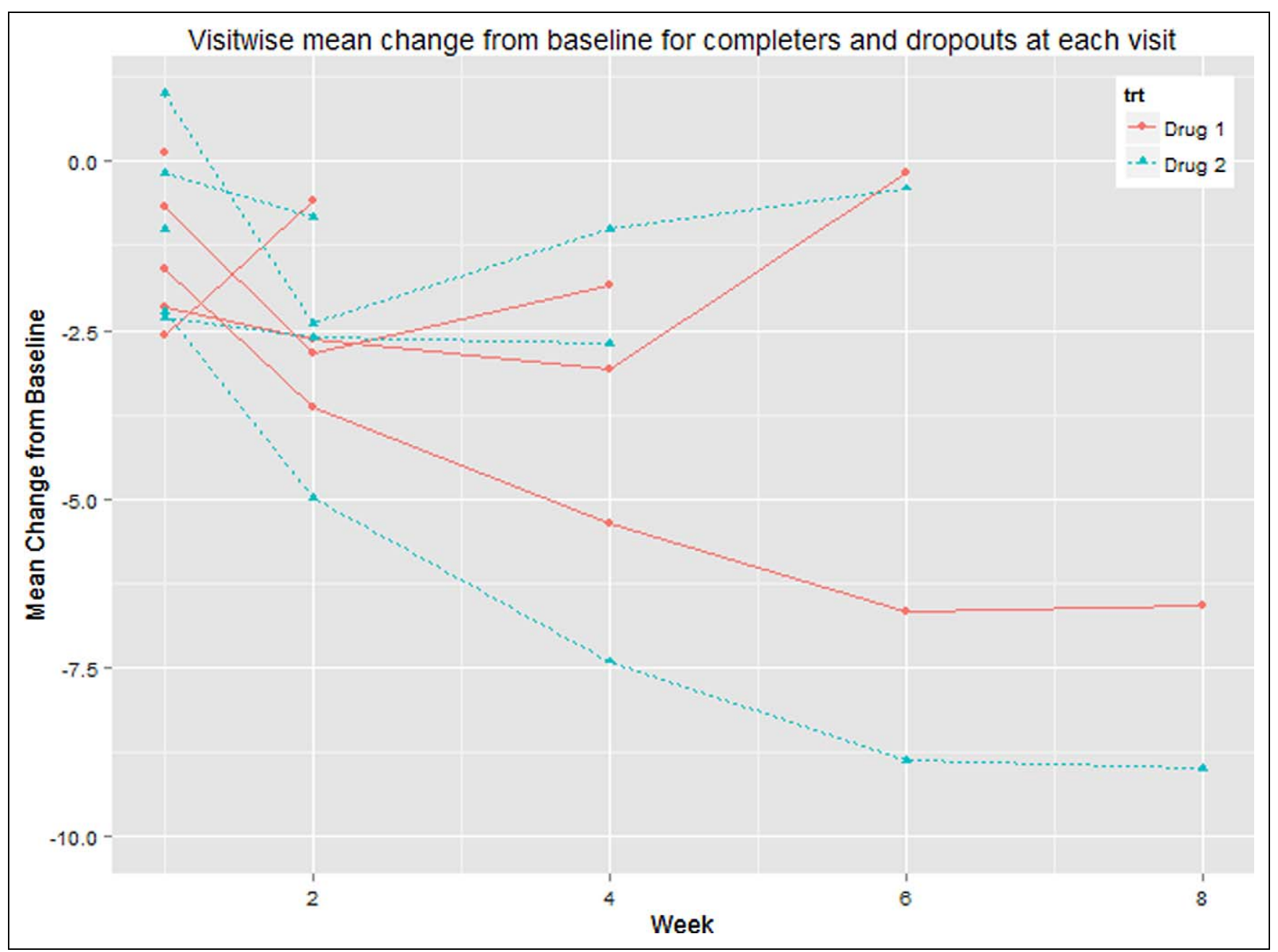

Figure 2. Visit-wise mean changes from baseline in $\mathrm{HAMD}_{17}$ for patients who completed the trial versus those who discontinued early in the high dropout data set.

Table 3. Variance covariance and correlation matrices from primary analysis.

\begin{tabular}{|c|c|c|c|c|c|c|c|c|c|c|}
\hline \multirow[b]{2}{*}{ Week } & \multicolumn{5}{|c|}{ High Dropout } & \multicolumn{5}{|c|}{ Low Dropout } \\
\hline & I & 2 & 4 & 6 & 8 & I & 2 & 4 & 6 & 8 \\
\hline 1 & 20.16 & & & & & 7.22 & & & & \\
\hline 2 & 14.05 & 29.86 & & & & 4.79 & 15.50 & & & \\
\hline 4 & 11.55 & 19.74 & 32.09 & & & 3.87 & 11.54 & 18.07 & & \\
\hline 6 & 10.70 & 18.56 & 25.27 & 35.10 & & 3.74 & 9.96 & 14.65 & 20.59 & \\
\hline 8 & 11.51 & 17.67 & 22.57 & 30.88 & 39.55 & 2.23 & 7.03 & 10.73 & 16.61 & 22.82 \\
\hline 1 & 1.000 & & & & & 1.000 & & & & \\
\hline 2 & 0.573 & 1.000 & & & & 0.453 & 1.000 & & & \\
\hline 4 & 0.454 & 0.638 & 1.000 & & & 0.339 & 0.689 & 1.000 & & \\
\hline 6 & 0.402 & 0.573 & 0.753 & 1.000 & & 0.307 & 0.558 & 0.760 & 1.000 & \\
\hline 8 & 0.408 & 0.514 & 0.634 & 0.829 & 1.000 & 0.174 & 0.374 & 0.528 & 0.766 & 1.000 \\
\hline
\end{tabular}

be crossed with visit, as this is consistent with a final visit analysis of variance (ANOVA) when the data are complete. The imputation-based methods used later naturally focus on
ANOVA at final visit, so the results from this model are also included in Table 5 for comparison. The group means are shifted appreciably while the difference is altered only slightly. 
Table 4. Visit-wise LSMEAN and contrasts for $\mathrm{HAMD}_{17}$ from the primary analysis.

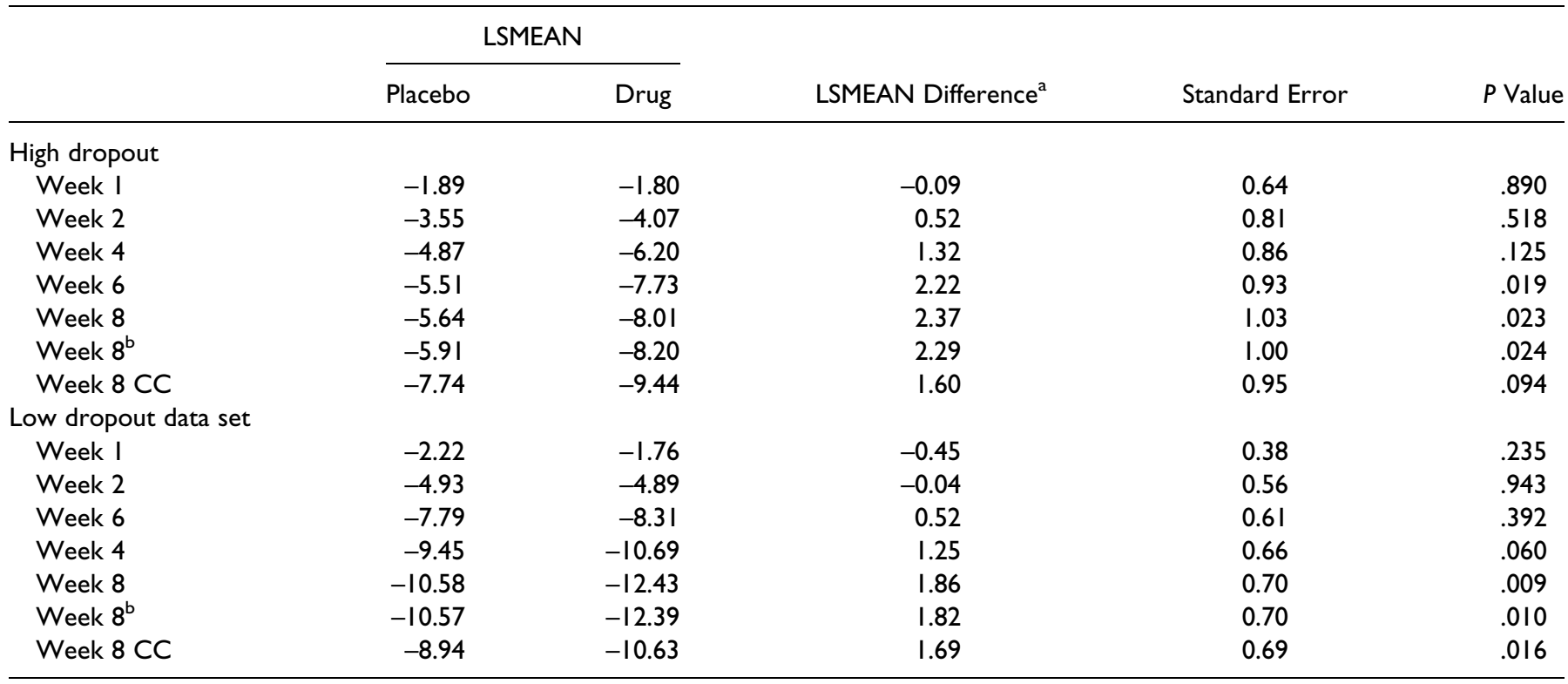

$\mathrm{CC}$, complete case analysis; $\mathrm{HAMD}_{17}$, Hamilton 17-item rating scale for depression; LSMEAN, least squares mean.

${ }^{a}$ Advantage of drug over placebo. Negative values indicate an advantage for placebo.

based on model with investigator-by-visit interaction.

Table 5. Results from selection model analyses of high dropout data set.

\begin{tabular}{|c|c|c|c|c|c|c|}
\hline 0.2 & 0.2 & -4.87 & -7.33 & 2.46 & 1.09 & .023 \\
\hline 0.0 & 0.2 & -5.60 & -7.38 & 1.78 & 1.05 & .091 \\
\hline-0.2 & 0.2 & -6.28 & $-7.4 I$ & 1.18 & 1.05 & .282 \\
\hline 0.0 & 0.0 & -5.63 & -8.00 & 2.37 & 1.04 & .022 \\
\hline-0.2 & 0.0 & -6.29 & -8.04 & 1.75 & 1.02 & .087 \\
\hline-0.4 & 0.0 & -6.75 & -8.05 & 1.30 & 1.02 & .204 \\
\hline 0.2 & -0.2 & -4.97 & -8.57 & 3.60 & 1.06 & .001 \\
\hline 0.0 & -0.2 & -5.67 & -8.57 & 2.89 & 1.03 & .004 \\
\hline-0.2 & -0.2 & -6.31 & -8.59 & 2.29 & 1.01 & .024 \\
\hline-0.2 & -0.4 & -6.33 & -8.98 & 2.64 & 1.01 & .009 \\
\hline-0.4 & -0.4 & -6.78 & -9.01 & 2.22 & 1.01 & .027 \\
\hline
\end{tabular}

LSMEAN, least squares mean.

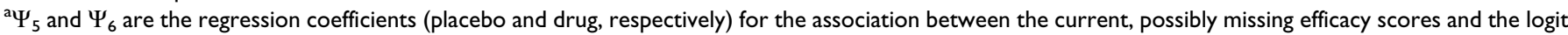
for probability of dropout.

${ }^{b}$ This combination of values is not plausible based on previous experience but is included for completeness of illustration.

The result of a complete cases analysis is also included for comparison only.

In the selection model analyses, the primary outcome was modeled by use of the repeated-measures model as in the primary analysis, and the probability of dropout was modeled with a logistic regression that fit the log odds of dropout as a function of a term for visit, separate intercepts $\left(\Psi_{1}, \Psi_{2}\right)$ for each treatment group, and separate linear regression coefficients for 
previous $\left(\Psi_{3}, \Psi_{4}\right)$ and current (possibly unobserved) efficacy outcomes $\left(\Psi_{5}, \Psi_{6}\right)$. Hence, the dependent variable from the measurement model was an independent variable in the dropout model. Fitting separate missingness models for each treatment allowed for different departures from MAR for drug and placebo groups.

The parameters $\Psi_{5}$ and $\Psi_{6}$ were of particular interest because they were the MNAR part of the model. Whenever possible, sensitivity analysis should be based on a predefined, plausible range of values for $\Psi_{5}$ and $\Psi_{6}$. These values are context sensitive and measure the increase in log odds for withdrawal for a single unit increase in the outcome measure. The range of values input for $\Psi_{5}$ and $\Psi_{6}$ was plausible based on previous experience from similar data. A value of 0.2 indicates that the odds of withdrawal increase by a factor 1.22 for each change of 1 unit on the HAMD 17 scale. However, pairing the largest value for $\Psi_{5}$ with the smallest value for $\Psi_{6}$ (and vice versa) resulted in combinations of values that were not plausible. These combinations are noted in Table 5. Setting $\Psi_{5}$ and $\Psi_{6}=0$ was an important validation because the missingness and outcome models become independent and the model reduces to the MAR one fitted as the primary analysis.

Across the range of plausible input values for $\Psi_{5}$ and $\Psi_{6}$ in the high dropout data set, the end point contrast ranged from 1.30 to 3.60 , which was a deviation of about \pm 1 around the MAR-based estimate of 2.37. The corresponding range in the low dropout data set was 1.71 to 2.01 , a much smaller deviation of \pm 0.15 around the MAR estimate of 1.86 . The reasons for greater variation in results from the high dropout data set are, in decreasing order of importance, (1) increased number of missing values; (2) larger variance leading to increased leverage for the same value of $\Psi$ in the missingness model; (3) stronger correlation between weeks, increasing the impact of withdrawals at early weeks; and (4) larger variance leading to increased potential impact of missing values on the mean.

Additional results from selection model analyses of the high dropout data set are summarized in Table 5. Results from the low dropout data set essentially agreed with those from the high dropout data set and are therefore not included for brevity.

As expected, when $\Psi_{5}=\Psi_{6}=0$, results matched results from the primary direct likelihood analysis. With equal negative values for $\Psi_{5}$ and $\Psi_{6}$, the within-group mean changes were greater than from equivalent MAR results. Conversely, identical positive values for $\Psi_{5}$ and $\Psi_{6}$ led to smaller within-group mean changes. The impact of a negative $\Psi$ value was to make those subjects with negative residuals more likely to withdraw, leading to reduced observed means. The selection model compensates by effectively increasing the LSMEAN to allow for this bias. If there is more dropout in the placebo group and $\Psi_{5}=\Psi_{6}<0$, the within-group mean changes are increased more in the placebo group than in the drug group and the
Table 6. Results from pattern-mixture model analyses of high dropout data set.

\begin{tabular}{lccc}
\hline $\begin{array}{l}\text { Identifying } \\
\text { Restriction }\end{array}$ & $\begin{array}{c}\text { End Point } \\
\text { Contrast }\end{array}$ & $\begin{array}{c}\text { Standard } \\
\text { Error }\end{array}$ & $P$ Value \\
\hline ACMV & 2.67 & 1.17 & .0224 \\
CCMV & 2.51 & 1.05 & .0166 \\
NCMV & 2.87 & 1.69 & .0895 \\
NFD-CCMV $(\Delta=+2)$ & 2.78 & 1.17 & .0180 \\
NFD-CCMV $(\Delta=-2)$ & 2.32 & 1.08 & .0320 \\
NFD-NCMV $(\Delta=+2)$ & 3.12 & 1.68 & .0634 \\
NFD-NCMV $(\Delta=-2)$ & 2.60 & 1.41 & .0645 \\
\hline
\end{tabular}

ACMV, available case missing values; CCMV, complete case missing values; NCMV, neighboring case missing values; NFD-CCMV, non-future dependence (in the spirit of CCMV); NFD-NCMV, non-future dependence (in the spirit of NCMV).

overall effect is to reduce the end point contrast. Conversely, with $\Psi_{5}=\Psi_{6}>0$, the end point contrast is increased. When the input values for $\Psi_{5}$ and $\Psi_{6}$ differed, between-group differences (end point contrasts) followed a consistent pattern dictated by the within-group changes. Whenever $\Psi_{6}$ (the regression coefficient for the drug group) was less than $\Psi_{5}$ (the regression coefficient for the placebo group), the treatment contrast was greater than from the MAR primary analysis; when $\Psi_{5}$ was less than $\Psi_{6}$, the treatment contrast was smaller than in MAR.

The separation of missingness models for each treatment group means that changing $\Psi_{5}$ has more impact on the placebo mean, while changing $\Psi_{6}$ has more impact on the drug group. However, some change is carried through to the other group, mostly driven by changes to the regression coefficients for baseline.

Results from pattern-mixture model (PMM) analyses under various identifying restrictions are summarized in Table 6 . The ACMV restriction assumed MAR, whereas CCMV, NCMV, and NFD assumed MNAR. Therefore, comparing the results under ACMV with those obtained under the other restrictions assessed the impact of departures from MAR. For NFD, the distribution of the current unobserved value, given the previous ones, is borrowed either from completers in NFD-CCMV or from the nearest pattern in NFD-NCMV.

Moreover, we introduced a shift of $\Delta= \pm 2$ in the location parameter of this distribution. PMM analysis requires that all effect parameters must be estimable in all patterns of dropouts. Consequently, for the analysis of the high dropout data set, the 6 centers have been pooled into 2 grouped centers, whereas the analysis of the low dropout data set was not feasible because the treatment effect was not estimable in several dropout patterns.

Reference-based imputations were done with a full multivariate repeated-measures model for parameter estimation including treatment group, pooled investigator, and baseline 
Table 7. Results from reference-based multiple imputation.

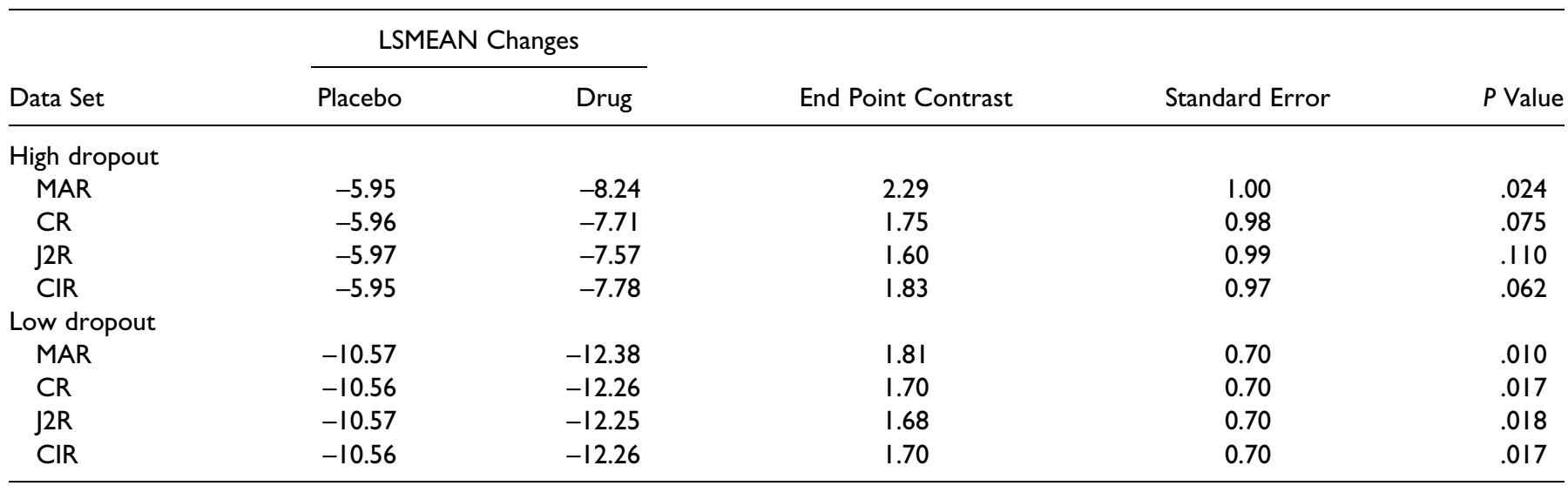

CIR, copy increment method; CR, copy reference; J2R, jump to reference; LSMEAN, least squares mean; MAR, missing at random.

score, all crossed with visit. The analysis model was ANOVA at week 8 with treatment, baseline, and pooled investigator in the model. The copy reference method of reference-based imputation was implemented with a multiple imputation model where all subjects were imputed as if they belonged to the placebo group. This causes a decay of any advantage gained from the treatment effect after early discontinuation of treatment. The speed of that decay is based on the correlation between the repeated measurements across visits. This approach was used to establish a plausibly conservative MNAR sensitivity analysis for testing robustness of the primary analysis to departures from MAR. However, this same numeric result was also used as a secondary assessment of effectiveness because it is reasonable to assume that treatment benefit declined over time after the medications were no longer taken.

Results from the reference-based imputations are summarized in Table 7. In the high dropout data set, the end point contrast from $\mathrm{CR}$ was $1.75(\mathrm{SE}=0.98, P=.075)$. In the low dropout data set, the end point contrast was 1.72 ( $\mathrm{SE}=0.70$, $P=.015)$. The results for other methods are included in Table 7. The MAR results concur with the direct likelihood when pooled investigator is crossed with time. As expected, the J2R results are more extreme than the CR results, while CIR is less extreme.

Progressive stress tests were implemented with a deltaadjustment controlled imputation approach based on the sequential regression algorithm. Delta-adjustments were applied to imputed values at all visits, but only for the drug group. The impact for a subject in the drug group withdrawing at week 2 is the buildup of an increment of approximately $\Delta \times$ 1 plus the sum of the correlation between week 2 and subsequent visits up to week 8 . That is, $\Delta \times 1+(0.51+0.63+$ $0.83+1)=2.97$ in the high withdrawal data set. This is in stark contrast with many of the other methods used earlier, especially NFD, where the impact of an early withdrawal is small compared with that of a late withdrawal. Here, an early withdrawal has a large impact compared with that of a late withdrawal.

Imputations were performed visit by visit, with patients' delta-adjusted imputed data contributing to imputed values at subsequent visits. The "tipping point" was identified by repeating the imputation process with progressively larger deltas.

Delta-adjustment stress test results are summarized in Table 8. In the high dropout data set, the delta had to be a worsening of 0.5 points on the $\mathrm{HAMD}_{17}$ in order to overturn the primary result (produce a nonsignificant result at 5\%). This was a change in end point contrast of 0.31 with a delta of 0.5 , which can be approximately explained as $0.5 *(9 * 2.97+$ $6 * 2.46+10 * 1.83+5 * 1) / 100=0.32$. The corresponding tipping point in the low dropout data set was 2.5 points with a shift of 0.41 , which is made up as $2.5 *(2 * 2.66+3 * 2.29$ $+2 * 1.77+1 * 1) / 100=0.42$.

Although the preceding results do not constitute a full sensitivity analyses, they do reveal some fundamental points about the 3 pillars of missing data. The primary analysis was specified to focus on a precisely defined efficacy estimand. A sensible primary analysis was specified, and sensitivity analyses aided understanding of the degree to which departures from MAR could alter inferences from the primary analysis. The sensitivity analyses were interpreted based on the properties of each approach rather than seen as simply another alternative approach. Most important, the results illustrated the benefit resulting from lower rates of missing data.

In sensitivity analyses of the low dropout data set, even the largest plausible departures from MAR had a relatively small impact on the treatment contrast. In the high dropout data set, sensitivity analyses generally supported the existence of a treatment effect; however, the possibility of departures from MAR severe enough to overturn the primary result, while not likely, could not be entirely ruled out. 
Table 8. Results from delta-adjustment multiple imputation-delta applied on all visits after discontinuation to active arm only.

\begin{tabular}{|c|c|c|c|c|c|c|}
\hline \multirow[b]{2}{*}{ Value of Delta Adj } & \multicolumn{3}{|c|}{ Low Dropout Data Set } & \multicolumn{3}{|c|}{ High Dropout Data Set } \\
\hline & End Point Contrast & Standard Error & $P$ Value & End Point Contrast & Standard Error & $P$ Value \\
\hline 0.5 & 1.77 & $0.7 \mathrm{I}$ & .013 & 2.00 & 1.03 & .051 \\
\hline 2.0 & 1.52 & 0.73 & .037 & & & \\
\hline 2.5 & 1.44 & 0.74 & .051 & & & \\
\hline
\end{tabular}

\section{Discussion}

Recent research has produced useful guidance for preventing and treating missing data. In the present paper, the 18 recommendations from the landmark NRC guidance ${ }^{3}$ were categorized into 3 pillars for successfully dealing with missing data: clearly specifying objectives and the associated causal estimands, minimizing missing data through trial design and conduct, and using sensible primary and sensitivity analyses. The NRC recommendations also pointed to needs for further research.

In response to the recommendations and needs, a Scientific Working Group was formed under the auspices of the Drug Information Association. The working group has developed standardized software tools for implementing sensitivity analyses that are freely available at www.missingdata.org.uk. The web site also provides additional details on the programs and how to use them.

Approaches and ideas presented in this paper are not intended as specific prescriptions for all trials. As the clinical contexts vary between studies, so too should the specific means of handling missing data.

Some authors argue that follow-up data collected after discontinuation of the initially randomized study drug and/or initiation of rescue medication should usually be included in the primary analysis. ${ }^{3}$ Others point to a more nuanced usage wherein follow-up data are often part of the primary estimand in outcome trials but not in symptomatic trials. ${ }^{4}$

Our view is that in many cases, both efficacy and effectiveness at the planned end point of the trial will be of interest because it is important to know both what happens when a drug is taken as directed (efficacy) and what happens when the drug is taken as in actual practice (effectiveness). The choice between efficacy and effectiveness as the primary estimand should be influenced by whether the trial design and conduct are more consistent with rigorously controlled efficacy assessments or more naturalistic effectiveness assessments. Whether follow-up data should be collected and/or included in the primary estimand can be considered on a case-by-case basis. However, given the confounding influences of rescue medications, the role for follow-up data in the analysis of symptomatic treatment trials would usually be secondary.
For longitudinal analyses of incomplete continuous data, consensus has emerged that a primary analysis based on MAR is often reasonable. Likelihood-based methods, MI, and wGEE are all useful MAR approaches whose specific attributes can be considered when tailoring a primary analysis to specific situations. With an MAR-based primary analysis, a focal point of sensitivity assessments is the impact of departures from MAR. Model-based MNAR methods such as selection models, pattern-mixture models, and shared-parameter models can be considered. Prior experience can guide analytic decisions such as plausible ranges of input values for selection models and appropriate identifying restrictions for pattern-mixture models.

Controlled-imputation methods can be especially useful in assessing the consequences of departures from MAR. If a plausibly conservative controlled imputation analysis agrees sufficiently with the primary result, the primary result can be declared robust to departures from MAR. Alternatively, a tipping point (progressive stress testing) format can be used to assess how severe departures from MAR must be in order to overturn conclusions from the primary analysis. If severe or implausible departures from MAR are required to negate the primary result, the primary result can be declared robust to departures from MAR.

We have focused on continuous end points and have not discussed categorical or time-to-event end points. The need for and means to clearly specify objectives and estimands, and to minimize missing data, apply regardless of type of end point. Obviously, specific analytic considerations will vary. The transition from continuous to categorical end points is relatively straightforward via a generalized linear mixed effects model framework where the issues of linearity and distribution can be addressed, with most of the missing data issues and sensitivity being similar to that of continuous data. More details can be found elsewhere. ${ }^{1}$ For time-to-event end points, a major challenge is to account for potential dependence between the failure and censoring times. Although detailed discussion of such analyses is beyond our present scope, the general approach of varying values of a parameter that describes this dependence has been advocated. ${ }^{3}$

We advocate the general framework of an MAR primary analysis supported by preplanned sensitivity analyses that 
include or perhaps even feature controlled imputations. However, the idiosyncratic nature of drug development in specific disease states mandates that development teams have early and explicit conversations with regulatory authorities to align on specific details of analyses and determine how sensitivity analyses will be used in overall inferences.

\section{Declaration of Conflicting Interests}

The author(s) declared no potential conflicts of interest with respect to the research, authorship, and/or publication of this article.

\section{Funding}

The author(s) received no financial support for the research, authorship, and/or publication of this article.

\section{References}

1. Mallinckrodt CH. Preventing and Treating Missing Data in Longitudinal Clinical Trials: A Practical Guide. New York, NY: Cambridge University Press; 2013.

2. European Medicines Agency. Committee for Medicinal Products for Human Use (CHMP). Guideline on missing data in confirmatory clinical trials. EMA/CPMP/EWP/1776/99 Rev. 1. http:// www.ema.europa.eu/docs/en_GB/document_library/Scientific_ guideline/2010/09/WC500096793.pdf. Published July 2, 2010.

3. National Research Council. The Prevention and Treatment of Missing Data in Clinical Trials. Panel on Handling Missing Data in Clinical Trials. Committee on National Statistics, Division of Behavioral and Social Sciences and Education. Washington, DC: The National Academies Press; 2010.

4. O'Neill RT, Temple R. The prevention and treatment of missing data in clinical trials: an FDA perspective on the importance of dealing with it. Clin Pharmacol Ther. 2012;91(3):550-554.

5. Molenberghs G, Kenward MG. Missing Data in Clinical Studies. Chichester, UK: John Wiley; 2007.

6. Mallinckrodt CH, Lane PW, Schnell D, Peng Y, Mancuso JP. Recommendations for the primary analysis of continuous end points in longitudinal clinical trials. Drug Inf J. 2008;42:305-319.

7. Mallinckrodt $\mathrm{CH}$, Kenward MG. Conceptual considerations regarding choice of end points, hypotheses, and analyses in longitudinal clinical trials. Drug Inf J. 2009;43(4):449-458.
8. Mallinckrodt CH, Lin Q, Lipkovich I, Molenberghs G. A structured approach to choosing estimands and estimators in longitudinal clinical trials. Pharm Stat. 2012;11:456-461.

9. Carpenter J, Roger J, Kenward M. Analysis of longitudinal trials with missing data: a framework for relevant, accessible assumptions, and inference via multiple imputation. J Biopharm Stat. In press.

10. Verbeke G, Molenberghs G. Linear Mixed Models for Longitudinal Data. New York, NY: Springer; 2000.

11. Fleming TR. Addressing missing data in clinical trials. Ann Intern Med. 2011;154:113-117.

12. Little R, Yau L. Intention-to-treat analysis for longitudinal studies with drop-outs. Biometrics. 1996;52(4):1324-1333.

13. Carpenter JR, Kenward MG. Missing data in randomised controlled trials - a practical guide. http://missingdata.1shtm.ac.uk/ downloads/rm04_jh17_mk.pdf. Published November 21, 2007. Accessed January 23, 2012.

14. Ratitch B, O'Kelly M. Implementation of pattern-mixture models using standard SAS/STAT procedures. http://pharmasug.org/pro ceedings/2011/SP/PharmaSUG-2011-SP04.pdf. Accessed October 4,2011 .

15. Teshome B, Lipkovich I, Molenberghs G, Mallinckrodt CH. Placebo multiple imputation: a new approach to sensitivity analyses for incomplete longitudinal clinical trial data. Journal of Biopharmaceutical Statistics. In press.

16. Ratitch B, O'Kelly M, Tosiello R. Missing data in clinical trials: from clinical assumptions to statistical analysis using pattern mixture models [published online January 4, 2013]. Pharm Stat. doi: 10.1002/pst.1549.

17. Goldstein DJ, Lu Y, Detke MJ, Wiltse C, Mallinckrodt C, Demitrack MA. Duloxetine in the treatment of depression: a double-blind placebo-controlled comparison with paroxetine. J Clin Psychopharmacol. 2004;24:389-399.

18. Detke MJ, Wiltse CG, Mallinckrodt $\mathrm{CH}$, McNamara RK, Demitrack MA, Bitter I. Duloxetine in the acute and long-term treatment of major depressive disorder: a placebo- and paroxetinecontrolled trial. Eur Neuropsychopharmacol. 2004;14(6):457-470.

19. Hamilton M. A rating scale for depression. J Neurol Neurosurg Psychiatry 1960;23:56-61.

20. SAS Institute Inc. SAS/STAT ${ }^{\mathbb{R}}$ 9.2. User's Guide. Cary, NC: SAS Institute; 2008. 